\title{
Biomaterials for bone regeneration
}

\author{
María-José Pérez-Sánchez ${ }^{1}$, Elena Ramírez-Glindon ${ }^{2}$, Miguel Lledó-Gil ${ }^{3}$, José-Luis Calvo-Guirado ${ }^{4}$, Cris- \\ tina Pérez-Sánchez ${ }^{5}$
}

${ }^{1}$ Dental surgeon. University of Murcia

${ }^{2}$ Dental surgeon. University of Murcia

${ }^{3}$ Associate Professor, Department of Adult Integrated Dentistry University of Valencia

${ }^{4}$ Professor of Adult Integrated Dentistry. University of Murcia. Manager, Postgraduate of Oral Implanthology. University of Murcia

${ }^{5}$ Dental surgeon. University of Murcia

Correspondence:

Avda/ General Ortin $N^{\circ} 3,2^{\circ} \mathrm{H}$

C.P. 30010 Ronda Sur

Murcia Spain

mjps7@hotmail.com

Received: 09/05/2009

Accepted: 28/11/2009

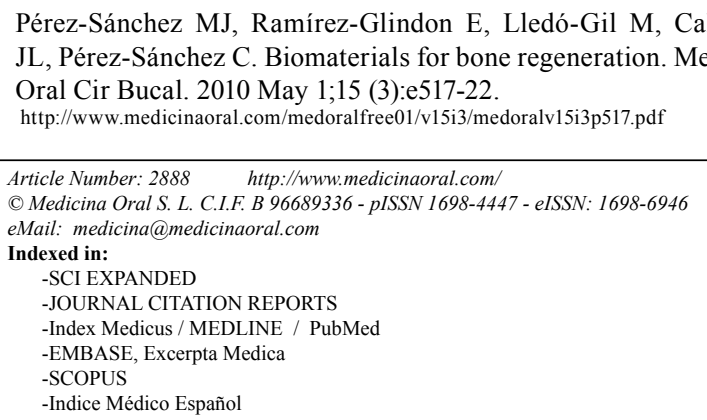

\begin{abstract}
Objective: This article reviews the literature on biomaterials used for bone regeneration.

Material and method: A total of seventeen bibliographic sources were found using the MEDLINE database and to avoid the variability of the search terms the thesaurus Mesh was used.

Results: These materials act essentially due to their osteoconductive ability, although their osteoinductive capacity is being improved with the use of growth factors. As to their effectiveness, many differences exist between them and some even affect bone regeneration negatively.

Conclusions: Biomaterials used for bone regeneration are valid when the correct material is used. As yet the osteogenic capacity of autogenous bone has not been equalled by biomaterials. Tissue engineering has caused great interest because of its many possibilities, although more studies are necessary in order to achieve the ambitious expectations when it comes to tissue or organ regeneration in the human body.
\end{abstract}

Key words: Biomaterials, bone regeneration, tissue engineering, growth factors, calcium phosphate, dental implants. 


\section{Introduction}

The life expectancy of the current population has increased considerably over recent decades due to improvements in the quality of life of individuals. One of the consequences of this phenomenon has been the increase in injuries and bone disease in older people who have a diminished bone regeneration capacity. Defects in oral and craniofacial tissues, resulting from trauma, tumours, infections, abnormal skeletal development or progressive deforming diseases, present a formidable challenge and restoration of these tissues is a subject of clinical, basic science and engineering concern $(1,2)$.

Bone is a highly vascularized and innervated connective tissue. It is composed of cells and mineralized organic matrix. Bone is the only body tissue capable of regeneration and remodelling process (it is in constant formation and resorption, this allows the renovation of $5-15 \%$ of the total bone mass per year under normal conditions). It is regulated by genetic, mechanical, vascular, hormonal, nutritional, and local factors. Amongst local factors, growth factors and cytokines, and recently the bone matrix proteins have been implicated as modulators of other local factors. Growth factors (GFs) are polypeptides produced by the bone cells themselves or in extra-osseous tissue, and act as modulators of the cellular functions, fundamentally growth, differentiation, and proliferation. The main GFs acting on the skeleton are IGF-I and II (insulin-like growth factor I and II), Transforming Growth Factor- $\beta$ (TGF- $\beta$ ), Bone Morphogenetic Proteins (BMP), Platelet-Derived Growth Factor (PDGF), Fibroblastic Growth Factor (FGF) and Vascular Endothelial Growth Factor (VEGF) (3-5).

Autografts have primarily been the material of choice to replace lost bone, although the use of autografts comes with some disadvantages such as the limited quantity available and its use also requires additional surgical procedures, and therefore, longer surgical time and possible complications of the wound of the donor site such as bleeding, pain and infection among others (1,2, 6-8). Allografts and xenografts have the potential of transferring pathogens $(2,9)$. To avoid the biological risk, these materials are subjected to exhaustive procedures which have dramatic effects primarily on their osteogenic and osteoinductive properties and these procedures can also reduce their structural integrity leading to graft fracture $(2,6,8)$. In order to minimize these complications, the alloplastic graft (synthetic bone graft substitute) has been developed. Synthetic bone graft possess both osteointegrative and osteoconductive properties $(6,10)$. The benefits of synthetic grafts include their availability, sterility and reduced morbidity (6). Long-term complications include stress shielding, loosening and mechanical or chemical breakdown of the material itself and they furthermore, lack the ability to adapt functionally (2). Synthetic grafts can be classified according to their chemical composition into four major categories: metallic implants such as titanium and stainless steel; ceramics such as calcium phosphate, alumina and glass; polymers such as polymethylmethacrylate and polyurethane and the fourth group is made up of composites, which are obtained after mixing several of the above (1).

The desire to create more biological alternatives has inspired the development of growth factors and tissue engineering $(1,2)$. The three main design components in tissue engineering are cell for osteogenesis, their extracellular matrix (scaffolds) for osteoconduction and a signalling system for osteoinduction, which can be used individually or in combination $(2,8)$.

Biomaterials were first developed in the 1960's and 1970 's. Currently the third generation of these materials is being developed so as to achieve bioactive and resorbable materials, capable of stimulating a specific cellular response at a molecular level (11).

\section{Material and Methods}

A search of published articles was carried out using documental or artificial language so that the words had no variability, so it was essential the choice of appropriate descriptors representative of the theme we were studying. We searched in the MEDLINE database produced by the National Library of Medicine in the United States. For this, one acceded by means of Internet to the link www. pubmed.gov. We used the Mesh thesaurus to introduce descriptors. We conducted a total of five bibliographic searches and a total of seventeen bibliographic sources were found. When we introduced "bone" in the Mesh we obtained numerous items. We selected Bone Regeneration y Bone Transplantation. We searched in PubMed for Bone Transplantation using the following inclusion criteria: articles published after July 2007, studies in adults between the ages of 19 and 44 and the articles had to be published in English or Spanish. Out of 559 articles 2 were selected $(9,12)$. Over 12,000 articles were found for Bone Regeneration. We carried out two types of bibliography searches. The first had the following inclusion criteria: full free text had to be available so as to obtain information immediately, they had to be published after July 2004 and in English or Spanish. Out of 244 articles 8 articles were selected $(3,4,7,10,13-16)$. In the second search, we looked for articles published after July 2008 so as to find the most recent information and these articles also had to be in English or Spanish. Out of 579 obtained 3 were selected $(5,8,17)$.

Oral Tissue Engineering was searched for in PubMed with the following inclusion criteria: articles published after July 2008 and again in English or Spanish. We obtained 190 articles of which only one was selected (2). Finally 3 of the reviewed articles, published after April 2001 and in English, were bibliographic references suggested by other dental surgeons $(1,6,11)$. 


\section{Results}

Of the articles selected in this review, we determined how many were purely theoretical and how many were both theoretical and practical, as shown in (Fig. 1), of the eight theoretical-practical articles two are in vitro, three in animals and three in humans.

In addition, articles were classified according to topic of study. The percentages are shown in (Fig. 2). The most numerous group compares different biomaterials and the smallest group contains tissue engineering articles. An important property which biomaterials must have so as to achieve optimal regeneration is their rate of degradation which should be similar to the rate of bone formation, that is to say, the mass of biomaterial must allow bone formation inside and persist until total bone replacement is achieved (2,7). Another interesting factor about the design of these materials is that the mass is typically maximized by maximizing porosity, where as mechanical properties are frequently maximized by minimizing porosity. One of the articles focuses on assessing the effect of the pore size of calcium phosphate scaffolds. Four pore sizes $(150,260,510,1220 \mu \mathrm{m})$ were used and they were evaluated at six, twelve and twentyfour weeks. The study showed that all the pore sizes

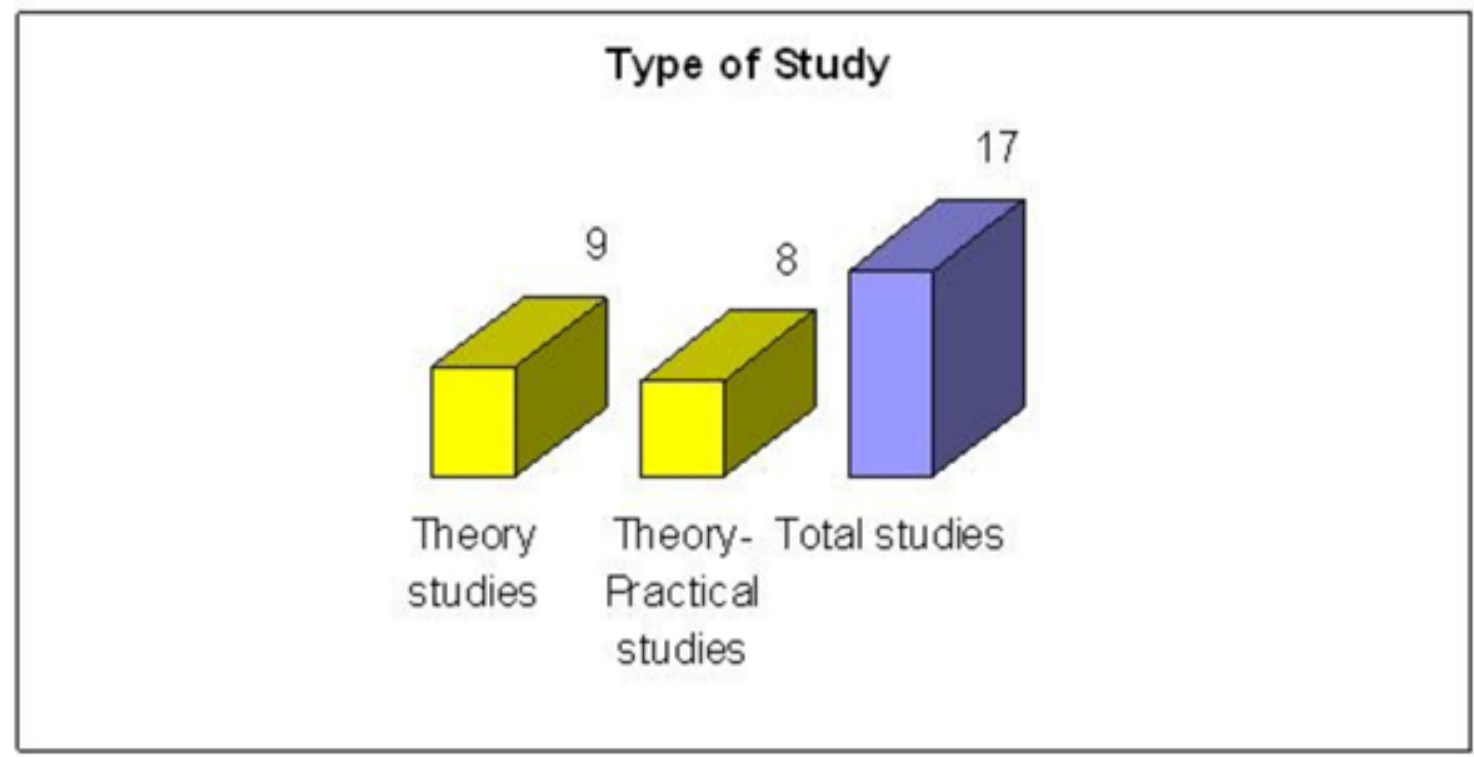

Fig. 1. Type of study.

\section{Topic of Study}

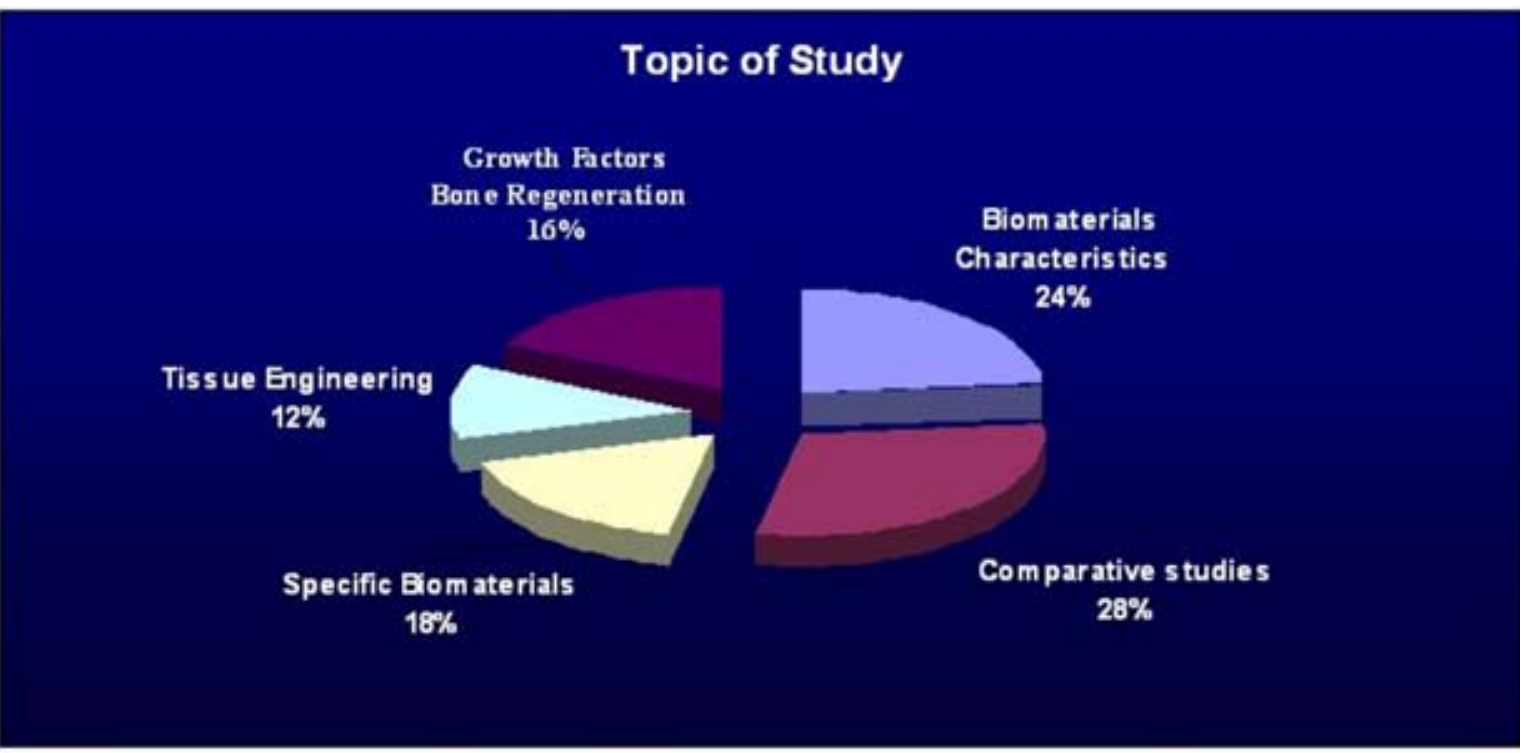

Fig. 2. Topic of study. 


\begin{tabular}{|c|c|c|c|c|c|c|c|c|c|c|c|c|}
\hline 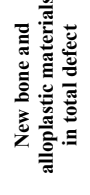 & I & I & 1 & 1 & 1 & 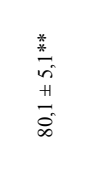 & $\begin{array}{l}\infty \\
\stackrel{+}{\infty} \\
\infty\end{array}$ & 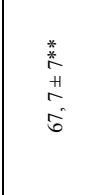 & 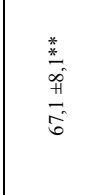 & 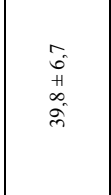 & 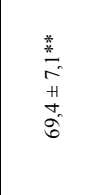 & $\tilde{\mathcal{H}}$ \\
\hline 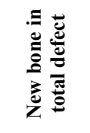 & I & I & 1 & 1 & 1 & 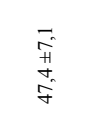 & $\begin{array}{l}\hat{a} \\
0 \\
0 \\
0 \\
0 \\
\text { r }\end{array}$ & 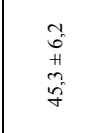 & $\begin{array}{l}m \\
a^{2} \\
+ \\
0 \\
\infty \\
\infty\end{array}$ & 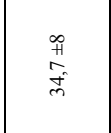 & $\begin{array}{l}\infty \\
\stackrel{+}{2} \\
\stackrel{n}{n}\end{array}$ & $\tilde{\mathcal{Z}}$ \\
\hline 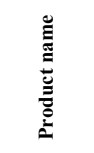 & I & I & 1 & 1 & 1 & $\begin{array}{l}\bar{E}_{\phi} \\
0 \\
\dot{0} \\
\dot{0}\end{array}$ & 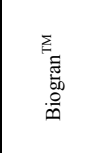 & 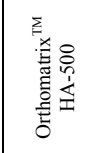 & 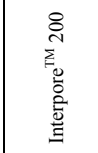 & 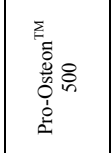 & 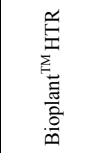 & I \\
\hline 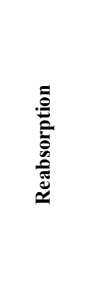 & 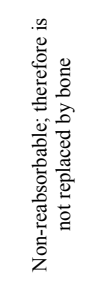 & 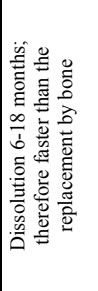 & 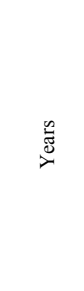 & 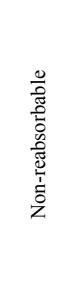 & 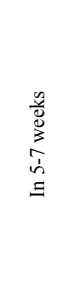 & 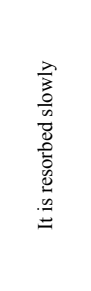 & 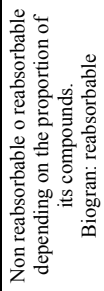 & 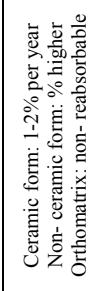 & 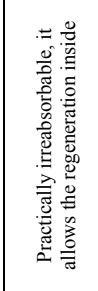 & 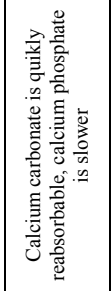 & 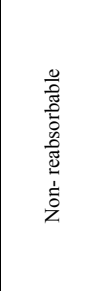 & I \\
\hline 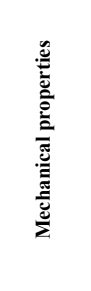 & 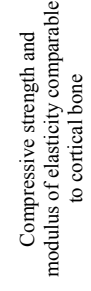 & 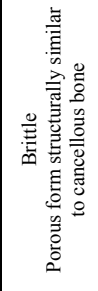 & 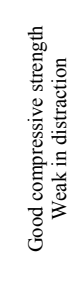 & 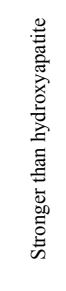 & 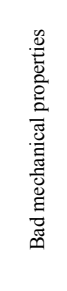 & 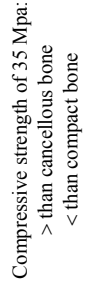 & 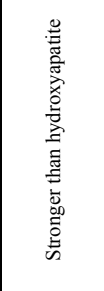 & 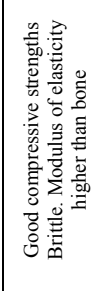 & 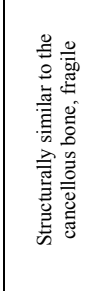 & 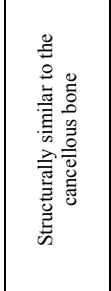 & 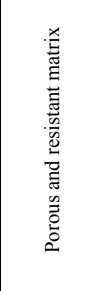 & I \\
\hline 言 & 衰 & 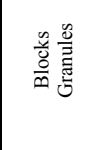 & $\frac{9}{8}$ & $\frac{8}{\frac{8}{8}}$ & 亭 & 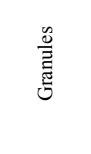 & 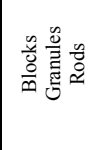 & 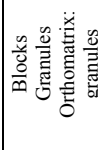 & 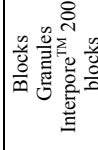 & $\begin{array}{l}\frac{v}{0} \\
\frac{0}{m}\end{array}$ & 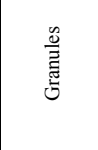 & I \\
\hline 氖 & 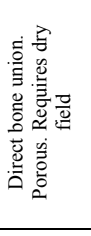 & 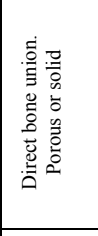 & 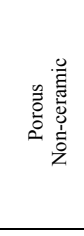 & 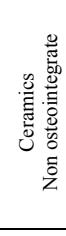 & 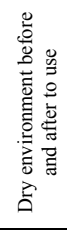 & 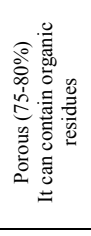 & 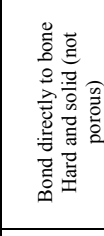 & 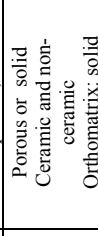 & 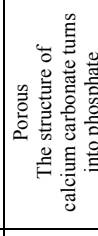 & 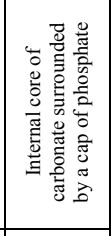 & 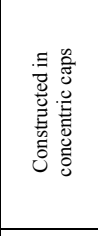 & I \\
\hline 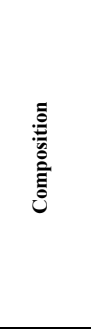 & 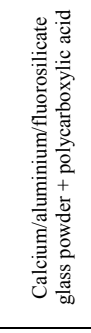 & 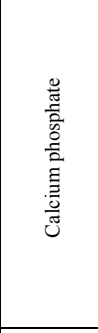 & 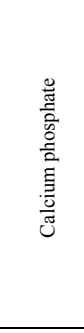 & 舀 & 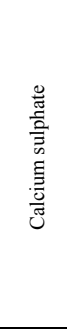 & 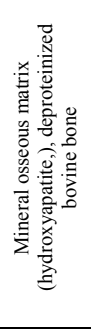 & 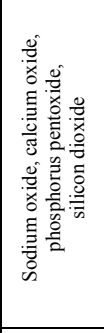 & 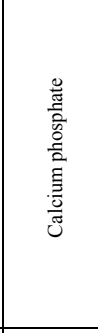 & 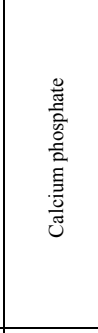 & 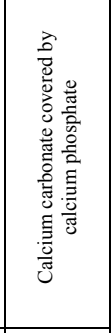 & 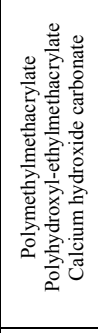 & I \\
\hline 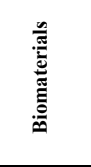 & 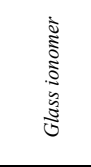 & 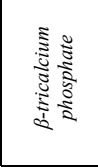 & 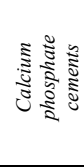 & 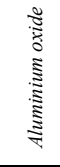 & 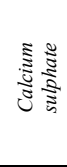 & 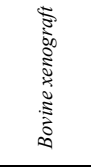 & 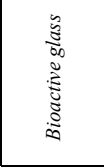 & 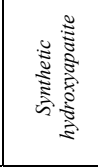 & 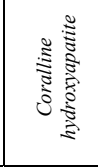 & 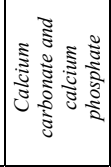 & 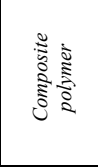 & \\
\hline
\end{tabular}


were biocompatible, osteoconductive and formed bone $(2,13)$.

Calcium phosphate appears to have osteoinductive qualities by ionic interaction with the environment surrounding it (1). Table 1 was prepared to demonstrate the most important characteristics of synthetic bone grafts and the great diversity of results obtained $(6,10,14)$.

In one of the articles reviewed, biomaterials were used to increase the alveolar ridge. The results varied depending on the biomaterial used, one of them even delayed the natural process of bone regeneration (7).

The application of biomaterials can be successful in the use of immediate implants. In one study a bioactive glass (PerioGlas ${ }^{\circledR}$ ) was joined with autogenous bone and blood of the patient. They found that this combination is suitable for use in fresh sockets around immediate implants. The success rate was $100 \%$ after twentyfour months of follow-up (15). In another study in which the surface of the implant was treated with bioceramics or ions, the rate of osteointegration was improved (17). When performing maxillary sinus lifts the use of biomaterials can also be useful. This is demonstrated in one of the articles reviewed where a mixture of autogenous bone and $\beta$-tricalcium phosphate was prepared. The sinus lift and implant placement were performed during the same surgical procedure. After an interval of twenty-seven to fifty-three months, no cases of implant failure were observed (12). Another material often used in these cases is a bovine hydroxyapatite, for example, Bio-Oss (14).

In another article biomaterials were successfully used for repairing surgically maxillary cystic bone defects. A bioactive glass reinforced with hydroxyapatite registered as Bonelike was used. All patients showed good bone regeneration after forty-eight weeks (9).

When considering tissue engineering, an ideal bone substitute should possess the following design requirements: a) biocompatibility, b) conductivity for attachment and proliferation of committed cells or heir progenitors and production of new extracellular matrix, c) ability to incorporate inductive factors to direct and enhance new tissue growth, d) support of vascular ingrowth for oxygen transport, e) mechanical integrity to support loads at the implant site, f) controlled, predictable, reproducible rate of degradation into non-toxic species that are easily metabolized or excreted and g) easy and cost-effective processing into irregular 3D shapes of sufficient size to fill clinically relevant defects (2).

GFs bind to target cell receptors and induce an intracellular signal transduction that reaches the nucleus and determines the biological response (5).

Bone Morphogenetic Proteins (BMPs) is the generic name of a family of proteins able to achieve the transformation of connective tissue into bone tissue, for which they are considered osteoinductive, therefore, are capable of inducing bone formation at ectopic sites.
Likewise, they are able to stimulate the differentiation of the stem cells towards different cell lines (adipose tissue, cartilage and bone). BMPs are not only present in the bone matrix, but can also be synthesized by cells of other lineages (e.g., macrophages). To date, 20 types and subtypes of BMPs have been reported. All BMPs belong to the TGF- $\beta$ superfamily except BMP-1. They are currently considered to be the most powerful factors in osteoblastic differentiation $(4,17)$.

Platelet-rich plasma (PRP) was proposed for use in oral and maxillofacial surgery, due to its high content of GFs. PRP can enhance regeneration mediated by the releasing of GFs, such as TGF- $\beta$, PDGF, and IGF-1, that are present inside of the $\alpha$-granules of platelets (17). Current sources for delivery of a GF mixture into the site of bone repair are platelet gel and demineralised bone matrix (17).

\section{Discussion}

It is interesting how Mah J et al. (10) and Schmitt SC et al. (16) when comparing different alloplastic bone grafts find great variability in their results. The studies differ in that the first was an in vivo study using rats, whilst the latter is carried out in vitro with similar cells to ovine osteoblast-like cells. The first study showed that with regards to the formation of new bone in total defect, there were no significant differences with the control group, but there were significant differences when particles of biomaterial in direct contact with the new formed bone were included (Table 1). If we look at the data from the second study, the sample where the largest growth occurred was in the cell culture without any biomaterial.

Other authors prefer to select a biomaterial and study it in a specific situation. This is the case for the study carried out by Masago $\mathrm{H}$ et al., who used PRP along with $\beta$-tricalcium phosphate granules, calcium phosphate cement powder and web form of titanium fibers (7).

Although many biomaterials have had a lengthy history of clinical success, very few interact with their surrounding host environment or promote integration with host tissue in an intelligent, proactive fashion. The desire for more biological approaches to biomaterial design that could yield materials that are more instructive to cells has led to an expansion and paradigm shift in the field of biomaterials. This is why GF, BMP, PRP and tissue engineering are the topic of study of many scientific investigations at this moment in time. These lines of investigation are based on the latest findings about biomaterials for bone regeneration. We found that the most recent articles were the ones that most considered these topics: Scheller EL et al., Devescovi V et al., Torroni A, and Avila G et al. $(2,5,8,17)$.

Some authors found that the association of biomaterials with autograft is beneficial for bone regeneration. This 
was the case for Aguirre Zorzano LA et al. (12), who associated autogenous bone with $\beta$-tricalcium phosphate. Gatti AM et al. (15) combined bioactive glass with the autogenous bone. Others prefer to associate different biomaterials as can be seen in a study carried out by Sousa $\mathrm{RC}$ et al., who used a composite (Bonelike ${ }^{\circledR}$ ) with a glass and hydroxyapatite base (9). Another line of investigation carried out by Avila $\mathrm{G}$ et al. was to apply 4 different substances to the implant surface (ceramics, bioactive proteins, ions and polymers) so as to improve bone regeneration (17). Scheller EL et al., Devescovi V et al. and Torroni A consider the biological mediators play an important role in improving bone regeneration $(2,5,8)$.

There is still much work needed and many questions to be resolved such as the way the biomaterial is applied (e.g. gel, foam or fibre), identification of appropriate cell sources for a desired application (e.g. autogenous versus allogenic cells; primary cells, cell lines, genetically modified cells versus stem cells; adult versus embryonic cells) o identification of spatial and temporal signals (e.g. growth factors, cytokines, chemokines) for tissuespecific differentiation and morphogenesis and the approach to deliver these signals (soluble versus insoluble; temporal and spatial control) represent design choices along the third axis of the tissue engineering triad (2). When the size of the lesion is larger than 6 to $9 \mathrm{~cm}$, the use of an autograft is not efficient and so the need for tissue engineering intensifies. Tissue engineering also comes with some drawbacks. The production of recombinant growth factors, collection and transport of the biopsies and culture of autologous cells are some of the factors that make tissue engineering time-, money- and labour-consuming. Another problem is that an engineered bone graft involves the inadequate vascularization of the inner portion of graft. This limits the formation of bone at the centre of the smaller grafts, and represents an insurmountable obstacle in the creation of larger grafts. The solution is to induce angiogenesis within the construct. Further improvement of biomaterials is needed in order to make tissue engineering more successful and, ideally, even unnecessary $(1,8)$.

Many variables have to be considered so as to achieve the best results in each individual clinical situation. For this a profound knowledge of biomaterials and their selection criteria is necessary as there are multiple possibilities available.

\section{Conclusions}

Currently, biomaterials are still far from ideal bone substitutes. They have certain disadvantages compared with autografts, because they lack the osteogenic and osteoinductive properties, but they have significant benefits in terms of availability, avoiding the risk of donor site complications and, of course, their well-developed osteointegrative and osteoconductive properties.
The studies seek to improve the characteristics of these bone substitutes. The two most important lines of research are based on one hand, on composites (combination of several biomaterials), and on the other hand, tissue engineering and growth factors. The latter emerges as an innovative, sophisticated and interesting field, because it is the nearest approximation to the autogenous graft, the only osteogenic material. Significant progress is expected in this young scientific field of biomaterials so as to achieve the needs of society and move towards a future of greater success.

\section{References}

1. Habibovic P, de Groot K. Osteoinductive biomaterials--properties and relevance in bone repair. J Tissue Eng Regen Med. 2007;1:25-32.

2. Scheller EL, Krebsbach PH, Kohn DH. Tissue engineering: state of the art in oral rehabilitation. J Oral Rehabil. 2009;36:368-89.

3. Fernández-Tresguerres-Hernández-Gil I, Alobera-Gracia MA, del-Canto-Pingarrón M, Blanco-Jerez L. Physiological bases of bone regeneration I. Histology and physiology of bone tissue. Med Oral Patol Oral Cir Bucal. 2006;11:E47-51.

4. Fernández-Tresguerres-Hernández-Gil I, Alobera-Gracia MA, del-Canto-Pingarrón M, Blanco-Jerez L. Physiological bases of bone regeneration II. The remodeling process. Med Oral Patol Oral Cir Bucal. 2006;11:E151-7.

5. Devescovi V, Leonardi E, Ciapetti G, Cenni E. Growth factors in bone repair. Chir Organi Mov. 2008;92:161-8.

6. Moore WR, Graves SE, Bain GI. Synthetic bone graft substitutes. ANZ J Surg. 2001;71:354-61.

7. Masago H, Shibuya Y, Munemoto S, Takeuchi J, Umeda M, Komori $\mathrm{T}$, et al. Alveolar ridge augmentation using various bone substitutes--a web form of titanium fibers promotes rapid bone development. Kobe J Med Sci. 2007;53:257-63.

8. Torroni A. Engineered bone grafts and bone flaps for maxillofacial defects: state of the art. J Oral Maxillofac Surg. 2009;67:1121-7.

9. Sousa RC, Lobato JV, Maurício AC, Hussain NS, Botelho CM, Lopes MA, et al. A clinical report of bone regeneration in maxillofacial surgery using bonelike synthetic bone graft. J Biomater Appl. 2008;22:373-85.

10. Mah J, Hung J, Wang J, Salih E. The efficacy of various alloplastic bone grafts on the healing of rat calvarial defects. Eur J Orthod. 2004;26:475-82.

11. Hench LL, Polak JM. Third-generation biomedical materials. Science. 2002;295:1014-7.

12. Aguirre Zorzano LA, Rodríguez Tojo MJ, Aguirre Urizar JM. Maxillary sinus lift with intraoral autologous bone and B--tricalcium phosphate: histological and histomorphometric clinical study. Med Oral Patol Oral Cir Bucal. 2007;12:E532-6.

13. Von Doernberg MC, Von Rechenberg B, Bohner M, Grünenfelder S, van Lenthe GH, Müller R, et al. In vivo behavior of calcium phosphate scaffolds with four different pore sizes. Biomaterials. 2006;27:5186-98.

14. Annalisa P, Furio P, Ilaria Z, Anna A, Luca S, Marcella M, et al. Anorganic bovine bone and a silicate-based synthetic bone activate different microRNAs. J Oral Sci. 2008;50:301-7.

15. Gatti AM, Simonetti LA, Monari E, Guidi S, Greenspan D. Bone augmentation with bioactive glass in three cases of dental implant placement. J Biomater Appl. 2006;20:325-39.

16. Schmitt SC, Wiedmann-Al-Ahmad M, Kuschnierz J, Al-Ahmad A, Huebner U, Schmelzeisen R, et al. Comparative in vitro study of the proliferation and growth of ovine osteoblast-like cells on various alloplastic biomaterials manufactured for augmentation and reconstruction of tissue or bone defects. J Mater Sci Mater Med. 2008;19:1441-50.

17. Avila G, Misch K, Galindo-Moreno P, Wang HL. Implant surface treatment using biomimetic agents. Implant Dent. 2009;18:17-26. 